\title{
Overview of EO-1, the First 120 days
}

\author{
Stephen G. Ungar \\ NASA, Goddard Space Flight Center \\ Biospheric Sciences Branch, Code 923 \\ Greenbelt, MD 20771
}

\begin{abstract}
The first 120 days of the EO-1 mission, constituting the accelerated mission, far exceeded the goal of acquiring sufficient data to insure meeting minimal EO-1 validation requirements. The strategy of using Southern Hemisphere test sites during the Northern Hemisphere winter solstice has left EO-1 with a rich legacy of data for a large variety of ecosystems under varying conditions.
\end{abstract}

\section{INTRODUCTION}

The Earth Observing One (EO-1) Satellite was launched from Vandenburgh Air Force Base on November 21, 2000. EO-1 is the first earth observing platform of NASA's New Millennium Program (NMP). The NMP develops new technologies and strategies for improving the quality of observations for NASA's future planetary and earth missions, while reducing cost and development time.

The EO-1 launch itself is an example of the NMP philosophy in action. EO-1 was co-manifested with SAC-C, an Argentine earth observing satellite, onboard a Delta-II rocket. This marks the first time that two major payloads of dissimilar nature were delivered to their respective orbits through a single Delta launch.

The EO-1 satellite contains three observing instruments supported by a variety of newly developed space technologies. The Advanced Land Imager (ALI) is a prototype for a Landsat-7 replacement instrument. The Hyperion Imaging Spectrometer is the first high spatial resolution imaging spectrometer to orbit the earth. The LEISA (Linear Etalon Imaging Spectral Array) Atmospheric Corrector (LAC) is an extremely high spectral resolution wedge imaging spectrometer, well suited to measuring atmospheric water vapor content. These instruments and their post launch performance are described at length in papers presented later in this session.

\section{THE EOS AM CONSTELLATION}

The EO-1 orbit is slightly to the east of Landsat-7, with an equatorial crossing time one minute later than that of Landsat-7. This results in EO-1 passing over the same groundtrack as Landsat-7, one minute later. The ground tracks of the various instruments compared to the Landsat-7 ground track is shown in Figure 1. Since EO-1 is a technology validation mission, it is extremely helpful to be able to make direct comparisons against the well-calibrated Landsat-7 sensor system. In a broader sense, EO-1 is not merely formation flying with Landsat-7, but joins a constellation of earth observing satellites (see Figure 2), including SAC-C, all exam- ining electromagnetic radiation along the same ground track with different swath widths and with a range of spatial and spectral resolution.

\section{MISSION DESIGN}

It is important to understand that EO-1 was built with flexibilities needed to validate the onboard technologies, rather than the costly redundancies and over-designed components required by a long time operational mission. The entire EO-1 validation mission data-gathering phase is currently scheduled to last one year. The satellite contains minimal redundancies and has a design life of less than two years, with. With this in mind, mission management adopted an "accelerated mission" approach to optimize the ability to validate the candidate technologies in the event of an early failure of any of the critical spacecraft components.

The roadmap for the first 120 days of operation consisted of approximately one week of spacecraft and rudimentary instrument checkout, followed by an intensive sixty-day instrument team checkout by the designers of the primary observing instruments. The instrument team continued acquiring images and performing tests during the second sixty days of the accelerated mission while the Science Validation Team (SVT) launched its Southern Hemisphere campaigns. These campaigns were designed to gather a sizable amount of data under conditions of relatively high solar illumination, for a large variety of landscapes representative of varying stages of vegetative development that are of global significance. These data will allow the SVT to exceed the minimal required validation criteria for the three observing systems in the event of an early critical component failure.

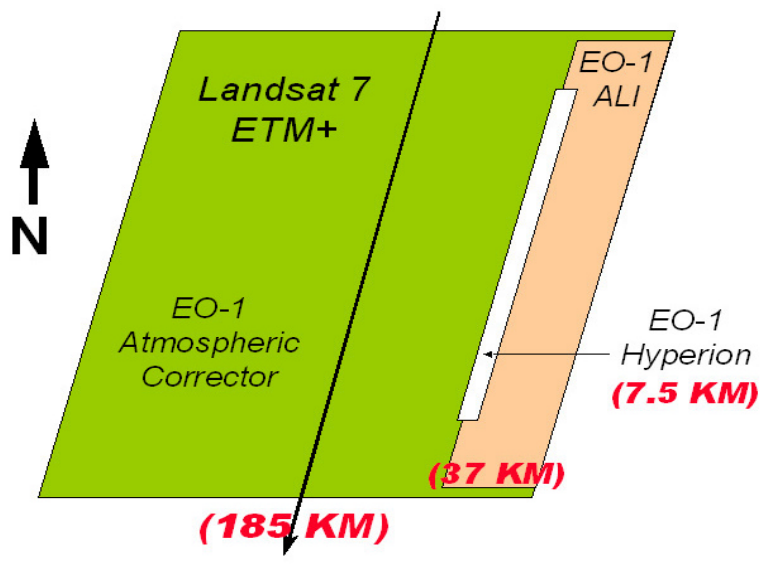

Fig. 1. EO-1 and Landsat-7 Instrument Ground Tracts 


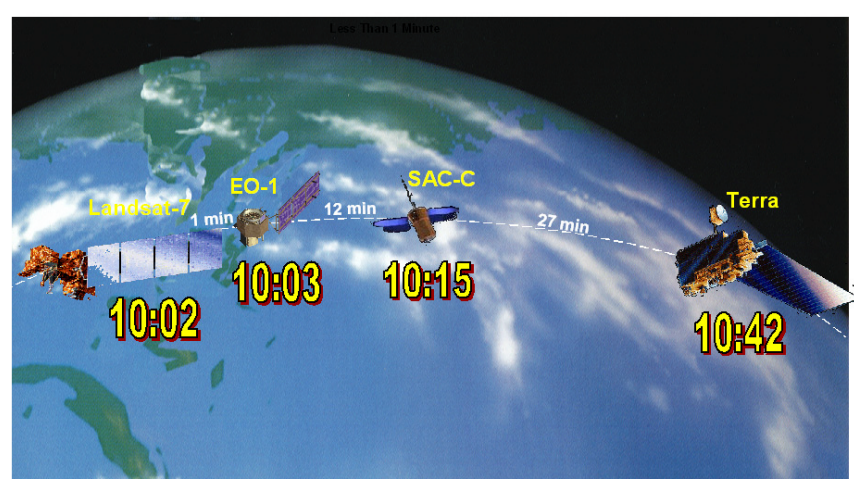

Fig. 2. The EOS AM Constellation

\section{SOUTHERN HEMISPHERE CAMPAIGNS}

An extensive field campaign was conducted in Argentina from early January through the third week of February. Argentina displays a very large diversity of ecosystem types, ranging from near tropical in the north to Antarctic in the south. There is an ample supply of rangeland, agricultural areas, forest, jungle, and semi-arid and arid regions. Figure 3 shows the location of eleven of the thirteen primary study areas selected for this campaign. Field measurements by U.S. and Argentine investigators were supported by AVIRIS over flights. Of particular note were two successful attempts to calibrate the Hyperion and ALI instruments by measurements made at Barreal Blanco (Zone 7) and Arizario (Zone 4), concurrent with EO-1 overpass. During the January $21^{\text {st }}$ overpass of EO-1 at Barreal Blanco, six teams of Argentine and US scientists made extensive ground measurements with a variety of spectrometers, photometers and other instruments, while the AVIRIS airborne imaging spectrometer simultaneously acquired data during low altitude flights aboard a Twin Otter. All EO-1 instruments were functional during this overpass with the exception of the SWIR bands on Hyperion. However, with the perfection of cryo-cooler operation later in the mission, all EO-1 sensor systems were fully operational for the calibration overpass at Arizario on February $6^{\text {th }}$. Two teams of US scientists equipped with multiple ASD spectrometers braved the four thousand meter elevation of this remarkably homogeneous dry-lake bed to make extensive ground measurements bolstered by AVIRIS over flights, contemporaneous with the EO-1 overpass. This calibration effort is highly significant in the annals of remote sensing calibrations since it is probably the highest altitude (minimal atmospheric perturbations) ever made for an earth observing system. The results of both of these calibrations have been factored into the EO-1 calibration.

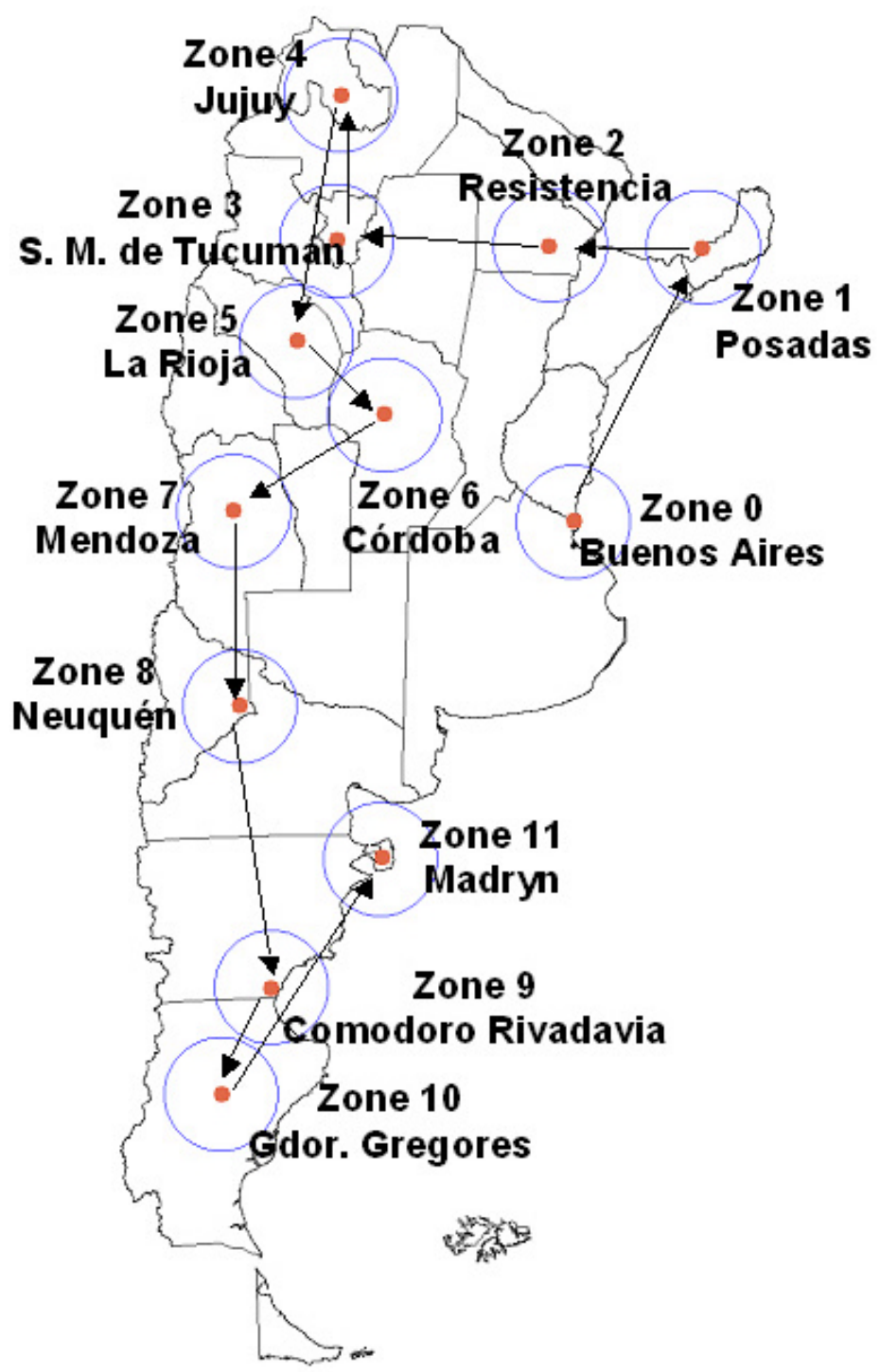

Fig. 3. AVIRIS Flight Zones for Argentina Test Sites

Extensive fieldwork, supported by HYMAP airborne spectral imager, was conducted in Australia from early launch throughout the first 120 days in orbit. The major areas of study are shown in Figure 4. Dr. David Jupp reports extensively on this activity elsewhere in the session. Members of the SVT made measurements at additional Southern Hemisphere sites in New Zealand and Africa. 


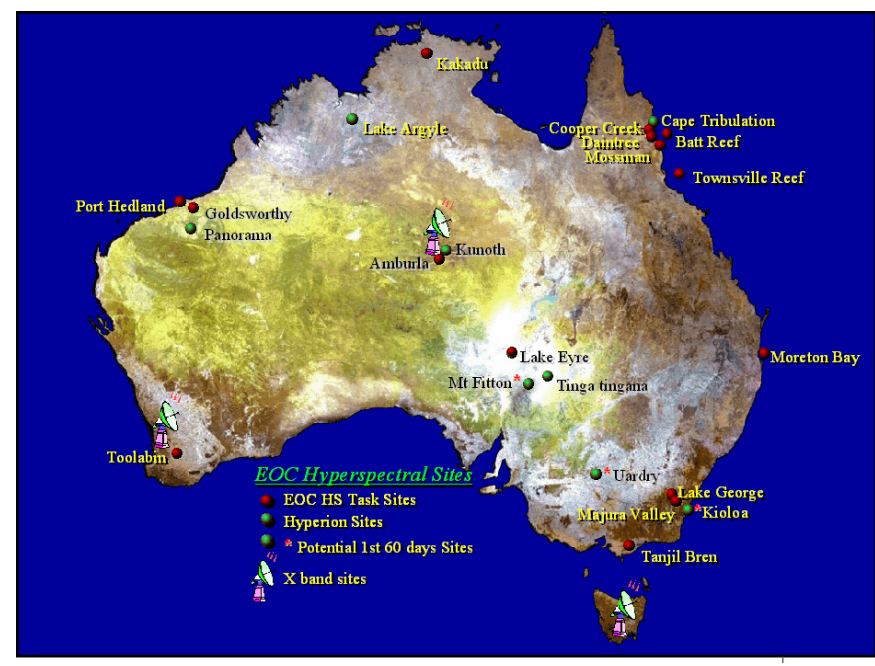

Fig. 4. Australian Campaign Test Site Locations

\section{CALIBRATION STRATEGY}

The primary mode of calibration for all EO-1 instruments is solar calibration wherein each of the three instruments views the sun through some sort of diffusing mechanism. The specific approach for each instrument will be described in detail for each of the instruments in specialized papers presented later in this session and in session B03AWE. When a solar calibration activity indicates apparent drifts in detector system characteristics, it is often difficult to distinguish between detector degradation and changes inherent in the solar calibration itself, such as degradation of the solar diffuser panel. The lunar calibration, which allows the detector system to view the lunar surface through the same optical path as when viewing the earth, can be used to measure overall detector system stability and thus will serve the role of solar diffuser monitor.

The operational flexibility of the EO-1 satellite has presented opportunities for carrying on many unique experiments to help quantitatively analyze the performance of each of the instruments. An active illumination experiment over Edwards Air Force Base, lunar views, planetary views, and stellar views have provided us with the ability to assess stray light performance as well as radiometric and geometric characteristics of ALI and Hyperion. Additionally stellar and planetary views (see Figures 5 and 6) offer the possibility of spectral characterization for Hyperion and LAC.

\section{CONCLUSION}

The Accelerated Mission has provided enough data to ensure meeting minimal EO-1 validation requirements. The Southern Hemisphere Campaigns provides data for a large variety of ecosystems under varying conditions. EO-1 will continue to collect data through the 2001 Northern Hemi- sphere growing season. The SVT will analyze and compare this data against Landsat-7, and other AM Constellation satellite borne observing systems, as well as airborne instruments and ground measurements. The manifold objectives of these analyses are to: (a) characterize and validate ALI as replacement for Landsat-7; (b) use Hyperion to explore space-borne spectral imaging as a means of more effectively accomplishing earth remote sensing objectives than can be done with multi-spectral observing systems; (c) characterize and validate the effectiveness and impact of removing atmospheric effects from Lansat-7 observation through use of LAC; (d) develop innovative ways to improve inter-satellite calibration; and (d) use formation flying to exploit potential synergisms among multiple satellite observing systems.

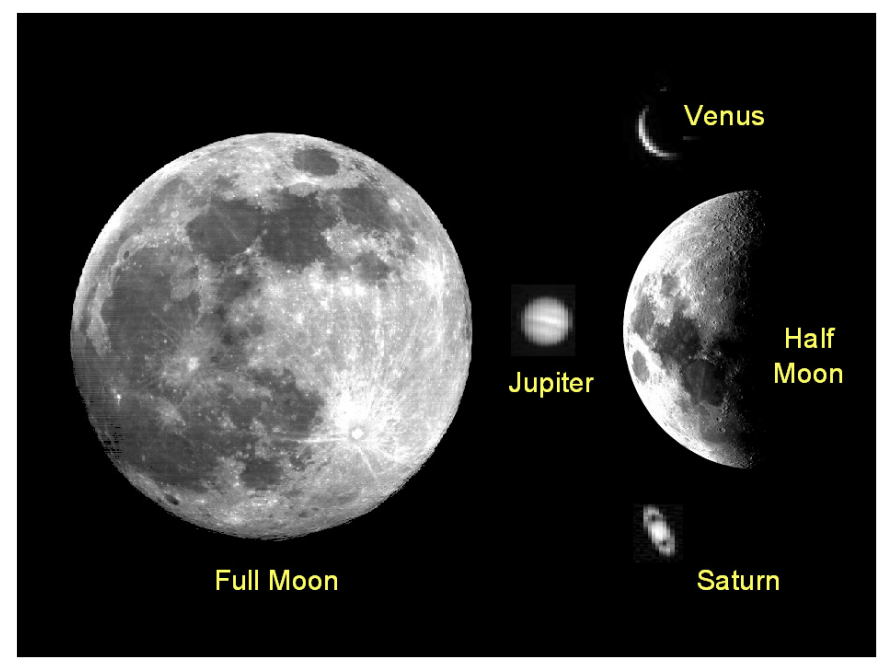

Fig. 5. Lunar and planetary views with ALI pan band

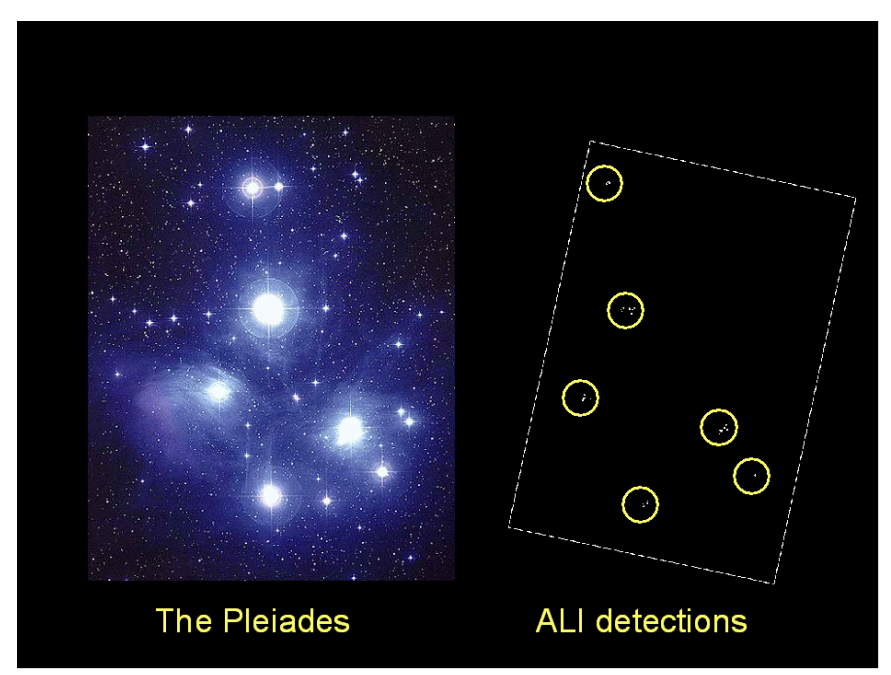

Fig. 5. The Pleiades star constellation as viewed with the ALI pan band Earth-based telescopic view to the left and EO-1 observations to right. 